\title{
Circulating MicroRNA-21 Is a Potential Diagnostic Biomarker in Gastric Cancer
}

\author{
Jianhong Wu, ${ }^{1}$ Guangxin Li, ${ }^{2}$ Zeyou Wang, ${ }^{3}$ Yongliang Yao, ${ }^{1}$ \\ Rui Chen, ${ }^{2}$ XiongYong $P u^{1}{ }^{1}$ and Jianjun Wang ${ }^{1}$ \\ ${ }^{1}$ Department of Clinical Laboratory, Kunshan First People's Hospital, Jiangsu University, Kunshan 215300, China \\ ${ }^{2}$ Department of Pathology, Chongqing Cancer Institute, Chongqing 400030, China \\ ${ }^{3}$ Institute of Cancer Research, Central South University, Changsha 410078, China
}

Correspondence should be addressed to Jianjun Wang; wangjianjun0520@163.com

Received 6 February 2015; Accepted 17 April 2015

Academic Editor: Olav Lapaire

Copyright (C) 2015 Jianhong Wu et al. This is an open access article distributed under the Creative Commons Attribution License, which permits unrestricted use, distribution, and reproduction in any medium, provided the original work is properly cited.

MicroRNA-21 was upexpressed in gastric cancer (GC) indicating that it is a potential diagnostic biomarker for GC. In this study, 50 GC patients and 50 healthy controls were recruited. miR-21 levels in serum and peripheral blood mononuclear cells (PBMCs) were quantified using quantitative real-time PCR. CA199, and CEA were measured using electrochemiluminescence assay. The sensitivity and specificity of circulating miR-21, CA199 and CEA in GC diagnosis, the correlation of circulating miR-21 to clinicopathological features, and the diagnostic value of miR-21 in different GC stages were determined. The levels of miR-21 in both serum and PBMCs increased significantly in GC patients comparing to healthy controls; however, no correlation was observed between circulating miR-21 level and clinicopathological features. The sensitivity and specificity of miR-21 in serum and PBMCs, and CA199 and CEA in GC diagnosis were $88.4 \%, 79.6 \%, 81.3 \%, 73.4 \%, 60.5 \%, 55.9 \%$, and $68.6 \%, 59.3 \%$, respectively. The positive prediction rates of circulating miR-21 in GC stages I to IV were all around 90\%, while those of CA199 and CEA were around or less than 50\%. Our data suggest circulating miR-21 (both in serum and in PBMCs) can serve as a good biomarker for GC and could be used in diagnosis of early (stage I) and late GC (stage IV).

\section{Introduction}

MicroRNAs (miRNAs), small single-strand RNA molecules with 18-25 nucleotides in length, possess the ability to modulate gene expression at posttranscription level $[1,2]$. Extensive research has revealed that miRNAs are involved in multiple biological processes including cell proliferation, differentiation, and apoptosis as well as development [3]. Among these identified miRNAs, many of them have demonstrated modulation in initiation and progression of various types of cancers [4-7].

miRNA-21 (miR-21), one of the first identified and most prevalent miRNAs in human cells, has been studied in various diseases including cardiovascular diseases as well as cancers. Particularly, since the miR-21-targeted genes identified till now are mostly tumor suppressors, miR-21 is closely related to various types of cancers including hepatocellular cancer [8], glioblastoma [9], glioma [10], and laryngeal carcinoma
[11] and has been designated as an oncomir [12-14]. Clinical research has revealed that the expression of miR-21 is elevated in a wide range of cancers including brain, breast, cervix, lung, liver, prostate, pancreas, and colon [15-22]. Due to the association with cancers, the potential of miR-21 as a cancer biomarker has also been widely studied for the past few years. In colorectal cancer, serum miR-21 could serve as a promising indicator for early detection as well as prognosis [23]. In colon adenocarcinoma, high level of miR-21 indicates poor therapeutic outcome and survival [24], whereas in lung cancer, serum miR-21 is diagnostic indicator with moderate sensitivity and specificity [25].

Gastric cancer (GC) is the second most common cancer around the world and is responsible for almost one million deaths per year worldwide. The high death rate is partially due to the lack of effective means for GC early screening. Cancer antigen 199 (CA199) and carcinoembryonic antigen (CEA) are two common tumor diagnostic markers; however, 
their specificity and sensitivity are too low for GC diagnosis. Therefore, a good biomarker of screening for GC is urgently needed. Previous studies have revealed that miR-21 has implications in GC progression. In vitro and ex vivo studies have shown that this microRNA is expressed in aberrantly high level in gastric cancer cell lines as well as primary tissues [26]. Moreover, miR-21 is associated with differentiation of tumor tissues as well as survival rates [26]. The mechanism study even discovered that miR-21 promotes GC proliferation and invasion probably by targeting PTEN [27]. However, the potential value of miR-21 as a screening biomarker in GC has not yet been investigated.

In the current study, by recruiting 50 GC patients and 50 healthy controls, we systematically evaluated the potential of circulating (serum and peripheral blood mononuclear cells) miR-21 as a screening GC marker in comparison to conventional cancer markers CA199 and CEA.

\section{Materials and Methods}

2.1. Ethical Statement. All protocols involving human subjects in the study were reviewed and approved by the Ethical Committee of Jiangsu University in accordance with the Declaration of Helsinki [28]. Informed written consents were obtained from the subjects who participated in this study.

2.2. Sample Collection. Fifty GC patients and 50 healthy individuals were recruited at the Department of Clinical Laboratory, Kunshan First People's Hospital, Jiangsu University, in 2014 from February to October. GC patients were classified into four stages according to Borrmann's classification [29]. For each participant, a total volume of $10 \mathrm{~mL}$ peripheral blood was collected. Five millilitres was mixed with anticoagulant for peripheral blood mononuclear cell (PBMC) isolation, while the other $5 \mathrm{~mL}$ without anticoagulant for serum isolation. Blood samples with anticoagulant were stored on ice and sent for PBMC isolation in $1 \mathrm{~h}$, while samples without anticoagulant were kept at room temperature and sent for serum isolation after coagulation.

2.3. Isolation of PBMCs and Serum Samples. PBMCs were isolated from whole blood samples using Ficoll-Paque Plus (GE healthcare) density gradient centrifugation according to the manufacturer's instructions. In brief, whole blood samples were layered on Ficoll-Paque Plus solution and centrifuged at $800 \mathrm{~g}$ for $30 \mathrm{~min}$ at $4^{\circ} \mathrm{C}$. PBMC layer was then extracted and washed with PBS twice and centrifuged at $350 \mathrm{~g}$ for $10 \mathrm{~min}$ at $4^{\circ} \mathrm{C}$. After washes, PBMCs were resuspended in $\mathrm{PBS}$, aliquoted, and stored at $-80^{\circ} \mathrm{C}$ till use. For serum isolation, coagulated blood samples were centrifuged at $1000 \mathrm{~g}$ for $10 \mathrm{~min}$ at $4^{\circ} \mathrm{C}$ and serum was collected and aliquoted and stored at $-80^{\circ} \mathrm{C}$ till use.

2.4. Total RNA Extraction and miR-21 Quantification by Quantitative Real-Time PCR ( $q R T-P C R)$. Total RNA was extracted from both serum and PBMCs using Trizol reagent (Invitrogen, Life Technologies) and the first strand cDNA was synthesized using PrimeScript RT Reagent Kit (Takara), both according to the manufacturer's instructions. miR-21 was quantified by qRT-PCR using U6 miRNA as control. The qRT-PCR was carried out using a SYBR Premix Ex Taq Kit (Takara) on a 7500 real-time PCR system (Applied Biosystems). The primers used for miR-21 amplification were (forward) $5^{\prime}$ ACGTTGTGTAGCTTATCAGACTG $3^{\prime}$ and (reverse) $5^{\prime}$ AATGGTTGTTCTCCACACTCTC $3^{\prime}$, and primers for U6 were (forward) $5^{\prime}$ ATTGGAACGATACAGAGAAGATT $3^{\prime}$ and (reverse) $5^{\prime}$ GGAACGCTTCACGAATTTG 3'. Each sample was determined in duplicate. The amplification specificity was validated by melting curve analysis and agarose gel electrophoreses of PCR products. miR-21 level was calculated relative to U6 miRNA using the $2^{-\Delta \Delta \mathrm{Ct}}$ formula, where $\Delta \Delta \mathrm{Ct}=\Delta \mathrm{Ct}_{\text {reference }}-\Delta \mathrm{Ct}_{\text {sample }}, \Delta \mathrm{Ct}$ is the difference in the cycling threshold between miR-21 and $\mathrm{U} 6, \Delta \mathrm{Ct}_{\text {sample }}$ is the Ct value of U6-normalized miR-21, and $\Delta \mathrm{Ct}_{\text {reference }}$ is the $\mathrm{Ct}$ value corresponding to control samples normalized to U6.

2.5. CEA and CA199 Measurements. The levels of CEA and CA199 in serum were determined using electrochemiluminescence assay with a Roche E170 MODULAR Immunoassay Analyzer according to the manufacturer's instructions (Roche).

2.6. Statistical Analysis. Mann-Whitney test was adopted for the comparison of miR-21 expression difference between GC patients and healthy controls. Kruskal-Wallis test was used for the comparisons between miR-21 and CA199 and CEA. Spearman correlation test was used for correlation analysis. Receiver operating characteristic (ROC) curve was carried out for the diagnostic evaluation of circulating miR-21 in GC and the cut-off values were determined using a training dataset and then applied to the remaining population. All analyses were performed with SPSS 16.0 software (SPSS Inc.) and a $p$ value $<0.05$ was considered statistically significant.

\section{Results}

3.1. Both Serum and PBMC miR-21 Levels Were Significantly Elevated in GC Patients in comparison to Healthy Controls. Circulating miR-21 levels in both serum and PBMCs were quantified by qRT-PCR using U6 as normalization control. First, U6 and miR-21 amplification curves as well as melting curves were analysed to check the validation of the current qRT-PCR system. As shown in Figures 1(a) and 1(b), no nonspecific products were amplified in the reaction, indicating that the primers used in the current qRT-PCR system could specifically amplify miR-21 and U6, respectively. Further analysis of U6 levels in serum and PBMCs from GC patients and healthy controls revealed that the expression of this conserved miRNA remained at comparable levels in both serum and PBMCs among all participants in this study, which indicated that U6 was an appropriate normalization control for miR-21 quantification in serum and PBMCs (Figure 1(c)).

Following qRT-PCR validation, we subsequently measured the miR-21 levels in serum and PBMCs from GC patients and healthy controls. As shown in Figure 1(d), basal level of miR-21 could be detected in both serum and PBMCs from healthy controls, while the level of this microRNA 


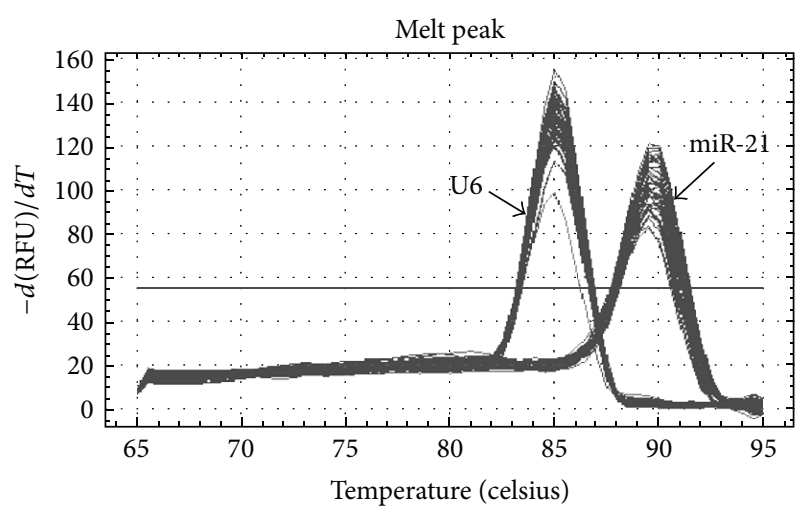

(a)

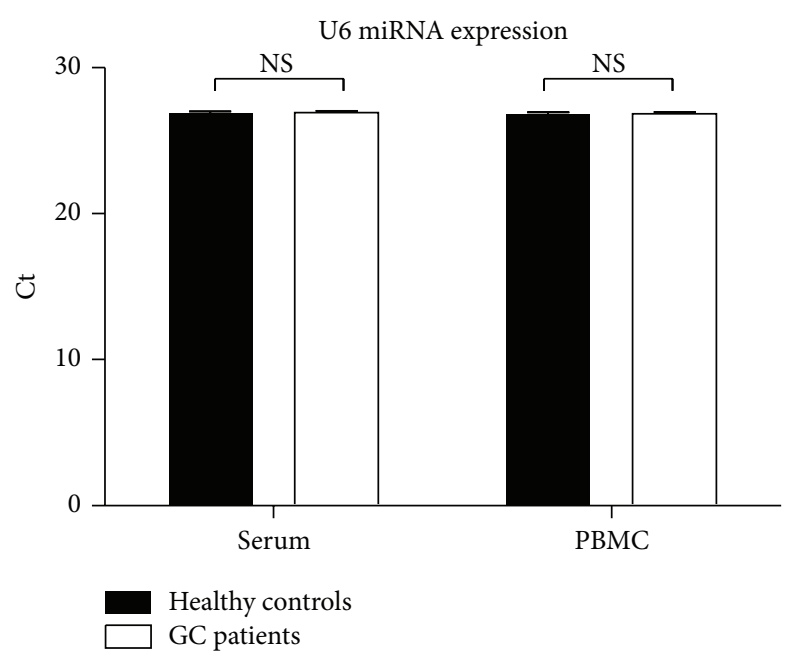

(c)

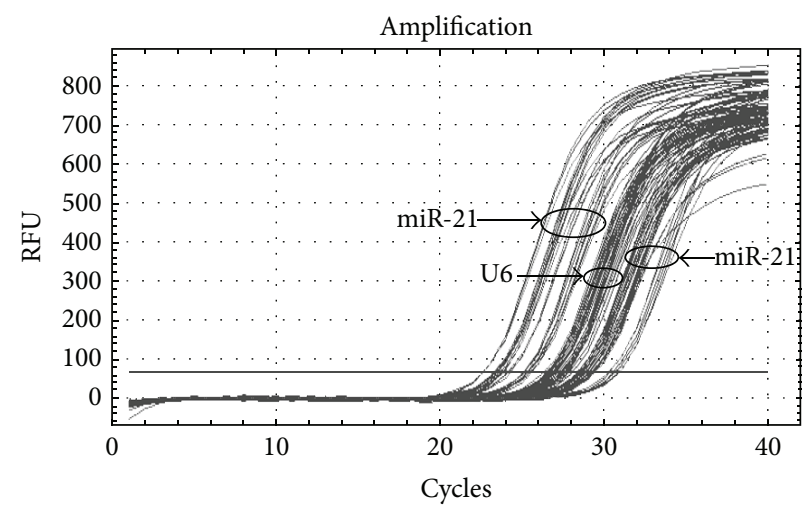

(b)

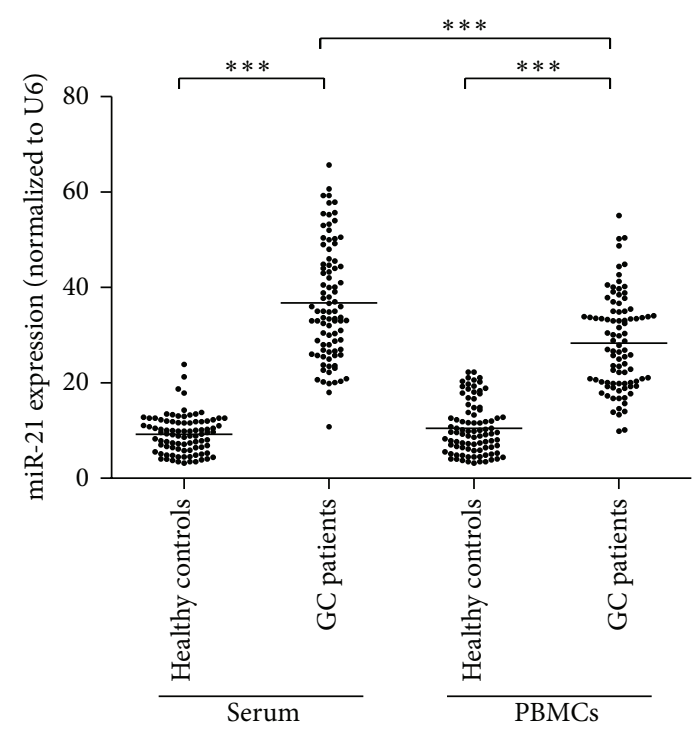

(d)

FIGURE 1: qRT-PCR quantification of miR-21 in serum and PBMCs of GC patients and healthy controls. (a) Amplification curves of miR-21 and U6. (b) Melting curves of miR-21 and U6. (c) Ct values of U6 in serum and PBMCS from GC patients and healthy controls. (d) miR-21 levels in serum and PBMCs of GC patients and healthy controls. NS: not statistically significant; ${ }^{* * *} p<0.001$.

was significantly increased in serum and PBMCs from GC patients $(p<0.001)$. Of note, the miR-21 elevation in serum was more profound than that in PBMCs $(p<0.001)$. Taken together, these results indicated that circulating (in serum and PBMCs) miR-21 elevation might be a concomitant clinical manifestation in GC disease.

3.2. miR-21 Level Was Not Associated with GC Clinicopathological Features. Subsequently, we further analysed the relationship of miR-21 with GC clinicopathological features. The analysis included clinical stage, age, gender, lymphatic metastasis, differentiation degree, and surgery history. Out of surprise, all the analysed variables, however, did not show any statistical correlation to miR-21 level in either serum or PBMCs $(p>0.05$ for all determinations, Table 1$)$.

3.3. Diagnostic Value of Circulating miR-21 in GC. Since the elevation of miR-21 in serum and PBMCs was related to GC, we next determined whether circulating miR-21 elevation could serve as a diagnostic biomarker for this cancer. The diagnostic value of circulating miR-21 was evaluated in comparison to conventional tumor biomarkers CA199 and CEA. First, ROC curve analysis was conducted to determine the specificity and sensitivity of circulating miR-21 in GC diagnosis. As shown in Figure 2, the area under curve (AUC) value of CA199 was 0.582 (95\% CI: 0.452-0.681), with the sensitivity of $60.5 \%$ and the specificity of $55.9 \%$ at the cut-off of 5.69. The AUC value of CEA was 0.667 (95\% CI: $0.536-$ 0.724 ), with the sensitivity of $68.6 \%$ and the specificity of $59.3 \%$ at the cut-off of 5.21. The AUC value of miR-21 in serum was 0.912 (95\% CI: $0.869-0.968$ ), with the sensitivity of $88.4 \%$ and the specificity of $79.6 \%$ at the cut-off of 2.78 . The AUC value of miR-21 in PBMCs was 0.898 (95\% CI: $0.838-0.935)$, with the sensitivity of $81.3 \%$ and the specificity of $73.4 \%$ at the cut-off of 3.02 . These data indicated that circulating (both in serum and in PBMCs) miR-21, but not 
TABLE 1: Association of miR-21 expression with clinicopathological features.

\begin{tabular}{|c|c|c|c|c|c|}
\hline Variable & $N$ & Serum miR-21 & $p$ & PBMC miR-21 & $p$ \\
\hline \multicolumn{6}{|c|}{ Clinical stage } \\
\hline I & 9 & $32.31(22.88-54.12)$ & \multirow{4}{*}{0.790} & $33.01(22.35-53.63)$ & \multirow{4}{*}{0.751} \\
\hline II & 11 & $31.62(21.14-50.12)$ & & $32.42(21.05-51.79)$ & \\
\hline III & 10 & $31.43(22.31-49.33)$ & & 31.38 (21.31-49.98) & \\
\hline IV & 18 & $30.82(23.45-41.23)$ & & $30.19(20.45-45.94)$ & \\
\hline \multicolumn{6}{|l|}{ Age } \\
\hline$<35$ & 8 & $31.77(23.28-51.02)$ & \multirow{4}{*}{0.812} & $30.33(22.43-50.78)$ & \multirow{4}{*}{0.892} \\
\hline $35-50$ & 10 & $29.67(23.21-50.53)$ & & $28.66(24.32-51.22)$ & \\
\hline $51-65$ & 18 & $30.25(24.22-52.38)$ & & 30.66 (23.99-51.59) & \\
\hline$>66$ & 14 & $32.81(25.88-53.62)$ & & $31.67(22.98-54.11)$ & \\
\hline \multicolumn{6}{|l|}{ Gender } \\
\hline Male & 24 & $29.95(23.24-49.38)$ & \multirow{2}{*}{0.844} & $28.66(22.42-48.78)$ & \multirow{2}{*}{0.789} \\
\hline Female & 26 & $30.17(22.66-50.19)$ & & $29.32(23.16-50.68)$ & \\
\hline \multicolumn{6}{|c|}{ Lymphatic metastasis } \\
\hline Yes & 27 & $29.99(25.87-51.12)$ & \multirow{2}{*}{0.678} & $31.54(24.55-51.66)$ & \multirow{2}{*}{0.643} \\
\hline No & 23 & $30.23(24.78-50.22)$ & & $32.11(24.11-50.35)$ & \\
\hline \multicolumn{6}{|c|}{ Differentiation degree } \\
\hline Low & 12 & $29.87(24.68-49.88)$ & \multirow{3}{*}{0.99} & $30.22(23.33-51.66)$ & \multirow{3}{*}{0.89} \\
\hline Middle & 17 & $30.19(24.98-50.86)$ & & $30.12(23.02-51.76)$ & \\
\hline High & 21 & $30.21(25.56-51.25)$ & & $31.01(24.45-51.87)$ & \\
\hline \multicolumn{6}{|l|}{ Surgery } \\
\hline Yes & 24 & $30.55(24.97-50.15)$ & \multirow{2}{*}{0.551} & $31.44(24.29-51.54)$ & \multirow{2}{*}{0.521} \\
\hline No & 26 & $29.78(23.99-51.21)$ & & $30.22(23.12-50.18)$ & \\
\hline
\end{tabular}

CA199 and CEA, had high odds in GC prediction. Further analysis by multivariate logistic regress confirmed that miR21 in both serum $(p<0.01)$ and PBMCs $(p<0.05)$ was good GC biomarker while the two conventional tumor biomarkers CA199 and CEA were not good indicators for GC ( $p>0.05$ for both determinations). Of note, although serum miR-21 demonstrated slightly higher sensitivity and specificity than PBMC miR-21 in ROC curve analysis, the pairwise comparisons of the two ROC curves revealed that there was no statistical difference between miR-21 in serum and PBMCs in GC prediction $(p>0.05)$.

We next determined whether circulating miR-21 could be used as an indicator in the diagnosis of GC in different stages. Positive prediction rates of miR-21 in serum and PBMCs as well as conventional tumor biomarkers CA199 and CEA were calculated and compared based on the cut-off values from ROC curves (Figure 2 and Table 2). Our results showed that the positive rate of CA199 in prediction of GC stages I to IV was $44.44 \%, 54.54 \%, 40.00 \%$, and $61.11 \%$, respectively, with an overall positive rate of $50.00 \%$. Similarly, the positive rate of CEA in prediction of GC stages I to IV was $33.33 \%, 45.45 \%$, $50.00 \%$, and $55.55 \%$, respectively, with an overall positive rate of $46.00 \%$. Unlike CA199 and CEA, the positive rates of miR21 in serum and PBMCs were considerably higher (stages I to IV and overall GC positive rates of serum miR-21 were $88.88 \%, 90.90 \%, 90.00 \%, 94.44 \%$, and $88.00 \%$ and of PBMCs miR-21 were $88.88 \%, 81.81 \%, 90.00 \%, 94.44 \%$, and $84.00 \%$ ). These data revealed that circulating miR-21 (both in serum and in PBMCs) was good biomarker for diagnosis of GC in all stages.

\section{Discussion}

With a total number of 700,000 GC-related deaths annually, GC has been the second most common cancer worldwide $[30,31]$. However, after decades of research, there is still a lack of effective biomarkers for GC diagnosis. For most cancers, blood-based proteins have been proven and widely used as biomarkers in clinical diagnosis. Unfortunately, situation is quite different for GC. Common tumor biomarkers like CA125, CA199, and CEA have exhibited poor diagnostic value in GC [32]. Consequently, the discovery of GC diagnostic biomarkers is in critical need. miRNAs, a group of regulatory small molecular RNAs, have been reported to be correlated with the progression of various cancers and some of them even have potential as biomarkers in cancer diagnosis [3335]. In the current study, our research discovered that circulating miR-21 (detected in serum and PBMCs) was significantly elevated in GC patients and could be a potential biomarker for GC diagnosis. Of note, our research also found that miR-21 as a biomarker was applicable for GC in all stages. Although it is beyond the scope of the current study, further research is warranted to investigate whether other miRNAs are also capable of indicating GC progression and more importantly whether miR-21 is the best choice among potential GC biomarkers. 


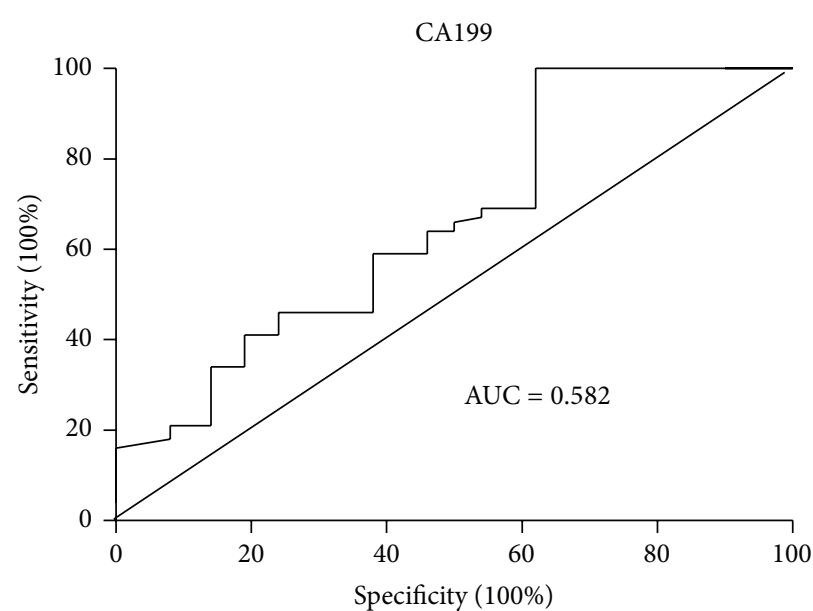

(a)

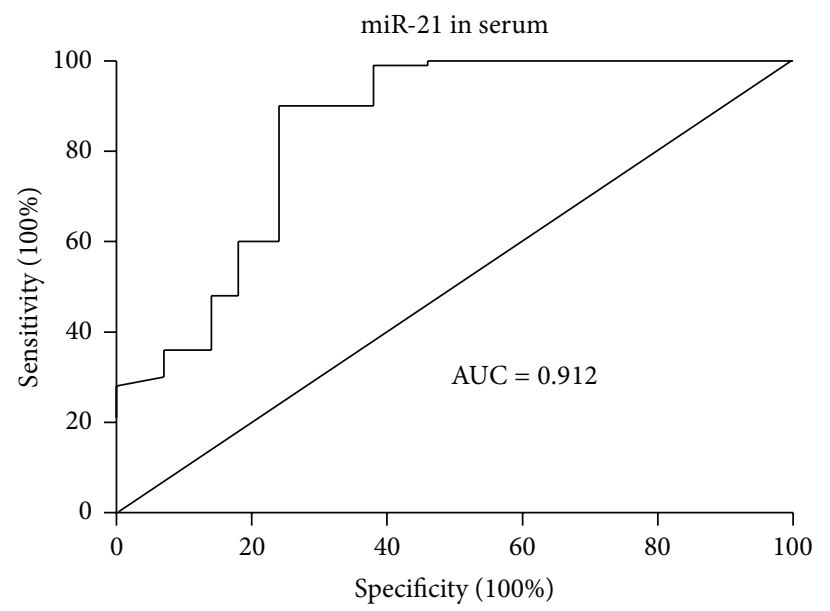

(c)

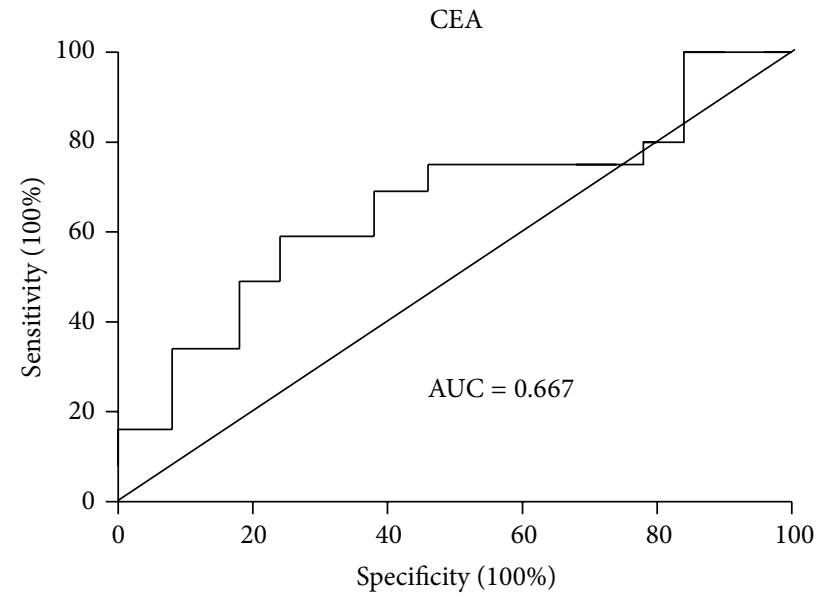

(b)

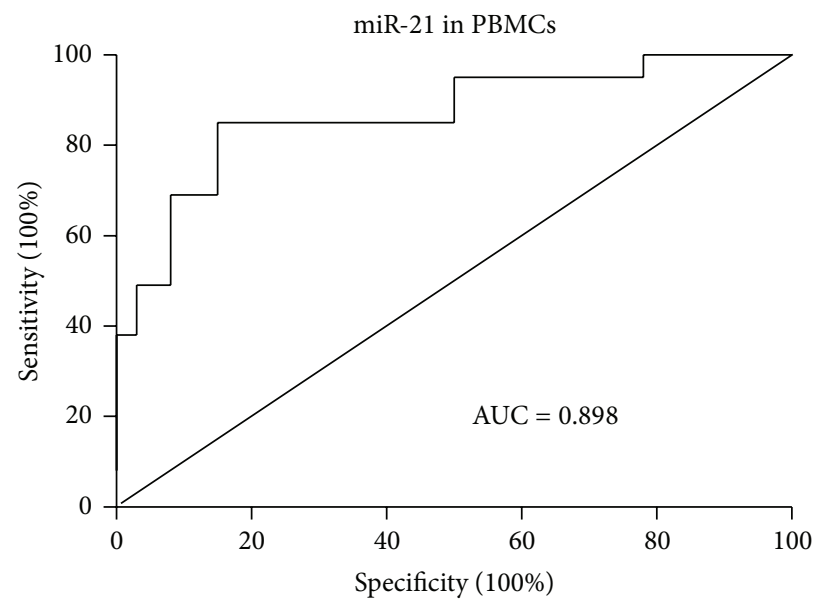

(d)

Figure 2: ROC analysis of CA199, CEA, and miR-21 in serum and PBMCs. An AUC value was given in each curve plot.

Diagnostic tests through noninvasive means are preferable, peripheral blood-based tests and therefore are widely used in clinical diagnosis. Circulating miRNAs as novel tumor biomarkers have demonstrated promising results in preclinical research and have been given great hope as measures for future cancer diagnosis. However, the aberrant elevation of miRNAs is originated from cancer tissues while some studies have suggested that miRNA expression profile was not identical to that observed in cancer tissues [36, 37]. Therefore, to what extent could the circulating microRNA level reflect the variation in cancer tissues is still needed to be further investigated in each type of cancers. In our study, miR-21 in blood is originated from GC tissues in GC patients, so further research is needed to determine the correlation of miR-21 levels between blood-based samples (e.g., serum and PBMCs) and GC tissues or gastric juice.

In addition, blood is a complex sample containing a variety of cells as well as many other components in the plasma. It is highly possible that levels of miRNAs in different blood components may be different and consequently reflect the miRNA variations in the cancer sites to distinctive extents
[38-40]. In the current study, we only focused on serum and PBMCs samples. Although miR-21 in both serum and PBMCs was proven to be potential biomarker for GC diagnosis, the elevation of miR-21 in serum, in comparison to that in PBMCs, was more profound and also demonstrated slightly better sensitivity and specificity in AUC analysis. The subtle difference of miR-21 in serum and PBMCs as GC diagnosis biomarkers discovered in our study could imply that there is a big chance that components other than serum and PBMCs (e.g., certain subclass of blood cells) may give even better results in GC diagnosis. Further research to address this mystery is warranted.

As a novel type of tumor biomarker, miRNA, in comparison to common protein biomarkers, has some unique characteristics. One difference with great importance is that miRNAs, albeit better than other RNAs in stability, are generally much less stable than proteins [41, 42]. Therefore, unlike protein-based tests, quantification of miRNAs may require extra measures to protect samples from degradation. In general, there are two ways to cope with this problem. One is to stabilize miRNAs in the sample and the other is to 


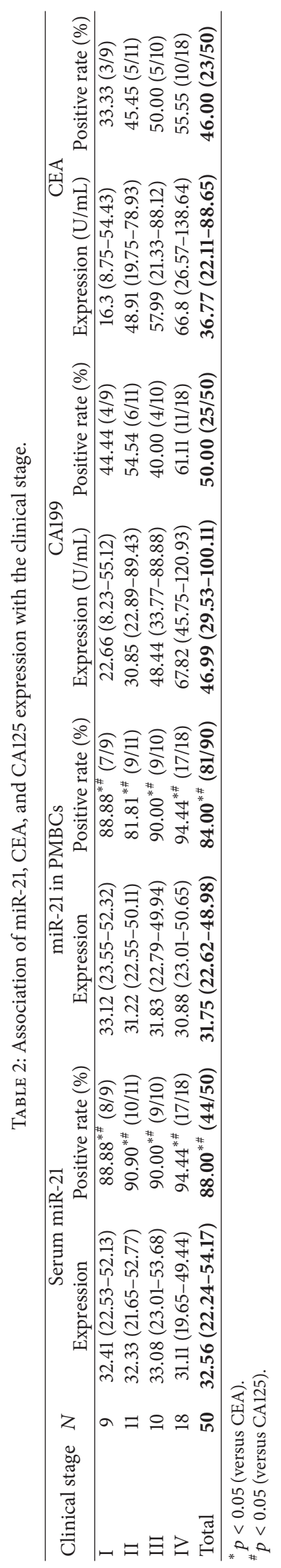


shorten time required for testing. In our study, to minimize miRNA degradation, samples were immediately processed for miRNA extraction after blood collection and stored at $-80^{\circ} \mathrm{C}$ before qRT-PCR and RNase inhibitors were added during PCR analysis. Previous studies by others have also described fast detection methods like microarray [43] and whole blood detection $[44,45]$ of miRNA. However, to define an optimal system for miRNA detection requires extensive research in the future [42].

In sum, based on a U6-controlled miR-21 qRT-PCR quantification method, our study indicated that circulating miR-21 (serum and PBMCs) was significantly increased in GC patients and could serve as GC diagnostic biomarkers. Moreover, the diagnostic value of miR-21 in GC was sustained from GC stages I to IV.

\section{Conclusions}

Circulating miR-21 (both in serum and in PBMCs) can serve as a good biomarker for GC and could be used in diagnosis of early (stage I) and late GC (stage IV).

\section{Conflict of Interests}

All authors declare that they have no financial or nonfinancial conflict of interests related to the subject matter or materials discussed in the paper.

\section{Authors' Contribution}

Jianhong $\mathrm{Wu}$ and Guangxin Li contributed equally to this work. Guangxin Li is also co-first author.

\section{Acknowledgments}

This work was supported by Science and Technology Project of Jiangsu Province in China (no. KS1347). The authors thank Xiangya Medical College for technical assistance.

\section{References}

[1] B. C. Schanen and X. Li, "Transcriptional regulation of mammalian miRNA genes," Genomics, vol. 97, no. 1, pp. 1-6, 2011.

[2] M. Lagos-Quintana, R. Rauhut, W. Lendeckel, and T. Tuschl, "Identification of novel genes coding for small expressed RNAs," Science, vol. 294, no. 5543, pp. 853-858, 2001.

[3] D. Sayed and M. Abdellatif, "Micrornas in development and disease," Physiological Reviews, vol. 91, no. 3, pp. 827-887, 2011.

[4] J. Krol, I. Loedige, and W. Filipowicz, "The widespread regulation of microRNA biogenesis, function and decay," Nature Reviews Genetics, vol. 11, no. 9, pp. 597-610, 2010.

[5] S. K. Shenouda and S. K. Alahari, "MicroRNA function in cancer: oncogene or a tumor suppressor?" Cancer and Metastasis Reviews, vol. 28, no. 3-4, pp. 369-378, 2009.

[6] E. Tili, J.-J. Michaille, V. Gandhi, W. Plunkett, D. Sampath, and G. A. Calin, "MiRNAs and their potential for use against cancer and other diseases," Future Oncology, vol. 3, no. 5, pp. 521-537, 2007.
[7] W. C. S. Cho, "OncomiRs: the discovery and progress of microRNAs in cancers," Molecular Cancer, vol. 6, no. 1, article 60, 2007.

[8] F. Meng, R. Henson, H. Wehbe-Janek, K. Ghoshal, S. T. Jacob, and T. Patel, "MicroRNA-21 regulates expression of the PTEN tumor suppressor gene in human hepatocellular cancer," Gastroenterology, vol. 133, no. 2, pp. 647-658, 2007.

[9] T. Papagiannakopoulos, A. Shapiro, and K. S. Kosik, "MicroRNA-21 targets a network of key tumor-suppressive pathways in glioblastoma cells," Cancer Research, vol. 68, no. 19, pp. 8164-8172, 2008.

[10] G. Gabriely, T. Wurdinger, S. Kesari et al., "MicroRNA 21 promotes glioma invasion by targeting matrix metalloproteinase regulators," Molecular and Cellular Biology, vol. 28, no. 17, pp. 5369-5380, 2008.

[11] M. Liu, H. Wu, T. Liu et al., "Regulation of the cell cycle gene, BTG2, by miR-21 in human laryngeal carcinoma," Cell Research, vol. 19, no. 7, pp. 828-837, 2009.

[12] T. X. Lu, A. Munitz, and M. E. Rothenberg, "MicroRNA-21 is up-regulated in allergic airway inflammation and regulates IL12 p35 expression," Journal of Immunology, vol. 182, no. 8, pp. 4994-5002, 2009.

[13] S. T. Hashimi, J. A. Fulcher, M. H. Chang, L. Gov, S. Wang, and B. Lee, "MicroRNA profiling identifies miR-34a and miR-21 and their target genes JAG1 and WNT1 in the coordinate regulation of dendritic cell differentiation," Blood, vol. 114, no. 2, pp. 404414, 2009.

[14] J. Zheng, H. Xue, T. Wang et al., "miR-21 downregulates the tumor suppressor P12 CDK2AP1 and stimulates cell proliferation and invasion," Journal of Cellular Biochemistry, vol. 112, no. 3, pp. 872-880, 2011.

[15] K. Wu, L. Li, and S. Li, "Circulating microRNA-21 as a biomarker for the detection of various carcinomas: an updated meta-analysis based on 36 studies," Tumor Biology, vol. 36, no. 3, pp. 1973-1981, 2015.

[16] J. A. Chan, A. M. Krichevsky, and K. S. Kosik, "MicroRNA-21 is an antiapoptotic factor in human glioblastoma cells," Cancer Research, vol. 65, no. 14, pp. 6029-6033, 2005.

[17] S. Volinia, G. A. Calin, C.-G. Liu et al., "A microRNA expression signature of human solid tumors defines cancer gene targets," Proceedings of the National Academy of Sciences of the United States of America, vol. 103, no. 7, pp. 2257-2261, 2006.

[18] A. Feber, L. Xi, J. D. Luketich et al., "MicroRNA expression profiles of esophageal cancer," The Journal of Thoracic and Cardiovascular Surgery, vol. 135, no. 2, pp. 255-260, 2008.

[19] O. Slaby, M. Svoboda, P. Fabian et al., "Altered expression of miR-21, miR-31, miR-143 and miR-145 is related to clinicopathologic features of colorectal cancer," Oncology, vol. 72, no. 5-6, pp. 397-402, 2008.

[20] E. J. Nam, H. Yoon, S. W. Kim et al., "MicroRNA expression profiles in serous ovarian carcinoma," Clinical Cancer Research, vol. 14, no. 9, pp. 2690-2695, 2008.

[21] M. V. Iorio, M. Ferracin, C.-G. Liu et al., "MicroRNA gene expression deregulation in human breast cancer," Cancer Research, vol. 65, no. 16, pp. 7065-7070, 2005.

[22] W.-O. Lui, N. Pourmand, B. K. Patterson, and A. Fire, "Patterns of known and novel small RNAs in human cervical cancer," Cancer Research, vol. 67, no. 13, pp. 6031-6043, 2007.

[23] Y. Toiyama, M. Takahashi, K. Hur et al., "Serum miR-21 as a diagnostic and prognostic biomarker in colorectal cancer," Journal of the National Cancer Institute, vol. 105, no. 12, pp. 849859, 2013. 
[24] A. J. Schetter, S. Y. Leung, J. J. Sohn et al., "MicroRNA expression profiles associated with prognosis and therapeutic outcome in colon adenocarcinoma," The Journal of the American Medical Association, vol. 299, no. 4, pp. 425-436, 2008.

[25] X. Yang, Y. Guo, Y. Du et al., "Serum microRNA-21 as a diagnostic marker for lung carcinoma: a systematic review and meta-analysis," PLoS ONE, vol. 9, no. 5, Article ID e97460, 2014.

[26] Z. Zhang, Z. Li, C. Gao et al., "MiR-21 plays a pivotal role in gastric cancer pathogenesis and progression," Laboratory Investigation, vol. 88, no. 12, pp. 1358-1366, 2008.

[27] B. G. Zhang, J. F. Li, B. Q. Yu, Z. G. Zhu, B. Y. Liu, and M. Yan, "microRNA-21 promotes tumor proliferation and invasion in gastric cancer by targeting PTEN," Oncology Reports, vol. 27, no. 4, pp. 1019-1026, 2012.

[28] W. M. Association, "Declaration of Helsinki, ethical principles for medical research involving human subjects," in Proceedings of the 52nd WMA General Assembly, Edinburgh, Scotland, 2000.

[29] K. Iriyama, T. Asakawa, H. Koike, H. Nishiwaki, and H. Suzuki, "Is extensive lymphadenectomy necessary for surgical treatment of intramucosal carcinoma of the stomach?" Archives of Surgery, vol. 124, no. 3, pp. 309-311, 1989.

[30] A. Jemal, F. Bray, M. M. Center, J. Ferlay, E. Ward, and D. Forman, "Global cancer statistics," CA: A Cancer Journal for Clinicians, vol. 61, no. 2, pp. 69-90, 2011.

[31] J. Ferlay, H.-R. Shin, F. Bray, D. Forman, C. Mathers, and D. M. Parkin, "Estimates of worldwide burden of cancer in 2008: GLOBOCAN 2008," International Journal of Cancer, vol. 127, no. 12, pp. 2893-2917, 2010.

[32] M. P. A. Ebert and C. Röcken, "Molecular screening of gastric cancer by proteome analysis," European Journal of Gastroenterology and Hepatology, vol. 18, no. 8, pp. 847-853, 2006.

[33] S. R. Singh and P. Rameshwar, MicroRNA in Development and in the Progression of Cancer, Springer, 2014.

[34] M. Ferracin and L. Lupini, "MicroRNA expression profiling and its clinical impact in breast cancer," in MicroRNAs: Key Regulators of Oncogenesis, pp. 355-367, Springer, 2014.

[35] T. A. Farazi, J. I. Hoell, P. Morozov, and T. Tuschl, "MicroRNAs in human cancer," in MicroRNA Cancer Regulation, vol. 774 of Advances in Experimental Medicine and Biology, pp. 1-20, Springer, Amsterdam, The Netherlands, 2013.

[36] S. S. C. Chim, T. K. F. Shing, E. C. W. Hung et al., "Detection and characterization of placental microRNAs in maternal plasma," Clinical Chemistry, vol. 54, no. 3, pp. 482-490, 2008.

[37] M. J. Lodes, M. Caraballo, D. Suciu, S. Munro, A. Kumar, and B. Anderson, "Detection of cancer with serum miRNAs on an oligonucleotide microarray," PLoS ONE, vol. 4, no. 7, Article ID e6229, 2009.

[38] G. M. Pasinetti, L. Ho, C. Dooley, B. Abbi, and G. Lange, "Select non-coding RNA in blood components provide novel clinically accessible biological surrogates for improved identification of traumatic brain injury in OEF/OIF Veterans," American Journal of Neurodegenerative Disease, vol. 1, no. 1, pp. 88-98, 2012.

[39] D.-J. Kim, S. Linnstaedt, J. Palma et al., "Plasma components affect accuracy of circulating cancer-related microRNA quantitation," The Journal of Molecular Diagnostics, vol. 14, no. 1, pp. 71-80, 2012.

[40] K. Wang, Y. Yuan, J.-H. Cho, S. McClarty, D. Baxter, and D. J. Galas, "Comparing the MicroRNA spectrum between serum and plasma," PLoS ONE, vol. 7, no. 7, Article ID e41561, 2012.

[41] S. Davis, S. Propp, S. M. Freier et al., "Potent inhibition of microRNA in vivo without degradation," Nucleic Acids Research, vol. 37, no. 1, pp. 70-77, 2009.
[42] J. S. Mcdonald, D. Milosevic, H. V. Reddi, S. K. Grebe, and A. Algeciras-Schimnich, "Analysis of circulating microRNA: preanalytical and analytical challenges," Clinical Chemistry, vol. 57, no. 6, pp. 833-840, 2011.

[43] W. Li and K. Ruan, "MicroRNA detection by microarray," Analytical and Bioanalytical Chemistry, vol. 394, no. 4, pp. 11171124, 2009.

[44] M. F. Corsten, R. Dennert, S. Jochems et al., "Circulating MicroRNA-208b and MicroRNA-499 reflect myocardial damage in cardiovascular disease," Circulation: Cardiovascular Genetics, vol. 3, no. 6, pp. 499-506, 2010.

[45] S. F. M. Häusler, A. Keller, P. A. Chandran et al., "Whole bloodderived miRNA profiles as potential new tools for ovarian cancer screening," British Journal of Cancer, vol. 103, no. 5, pp. 693-700, 2010. 


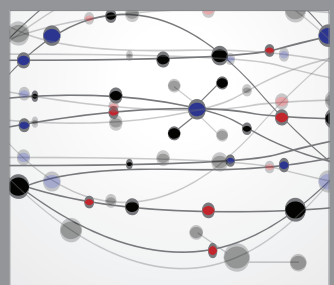

The Scientific World Journal
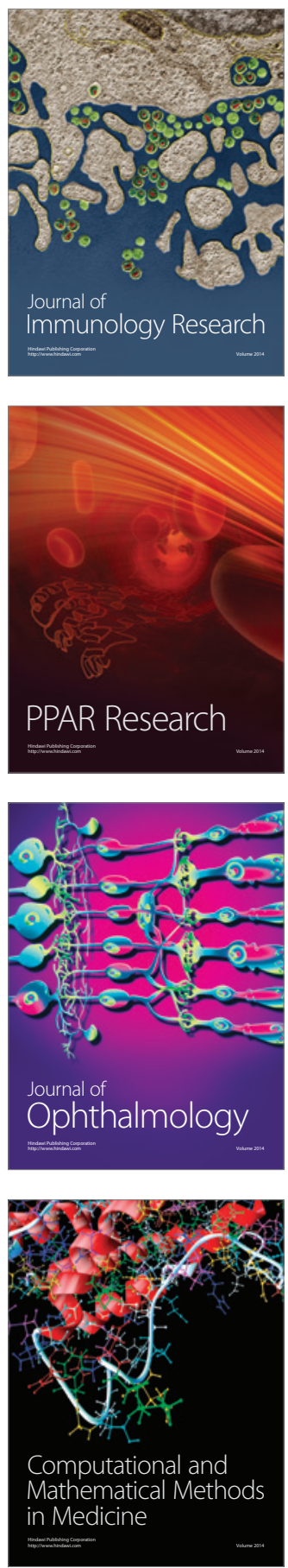

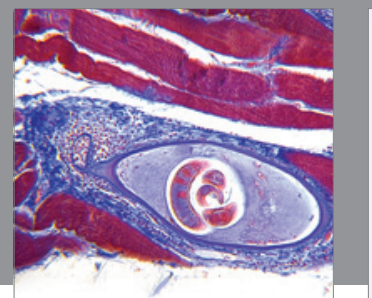

Gastroenterology

Research and Practice
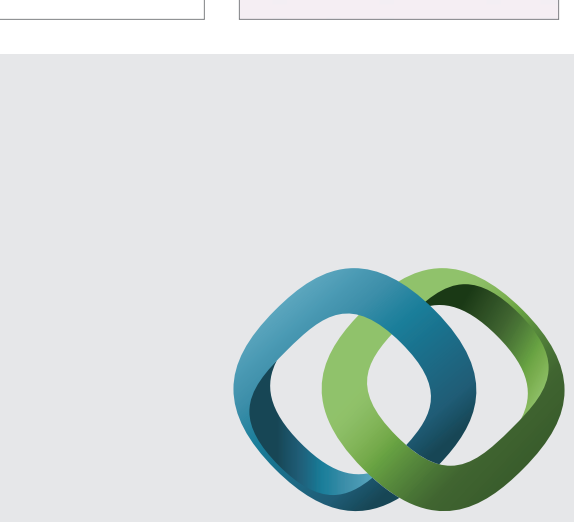

\section{Hindawi}

Submit your manuscripts at

http://www.hindawi.com
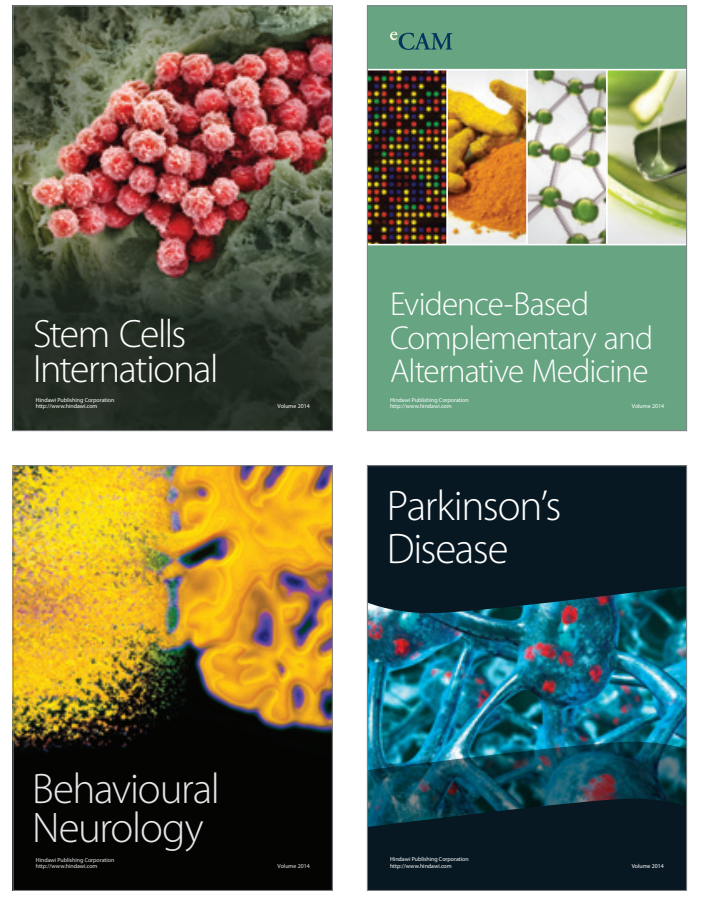
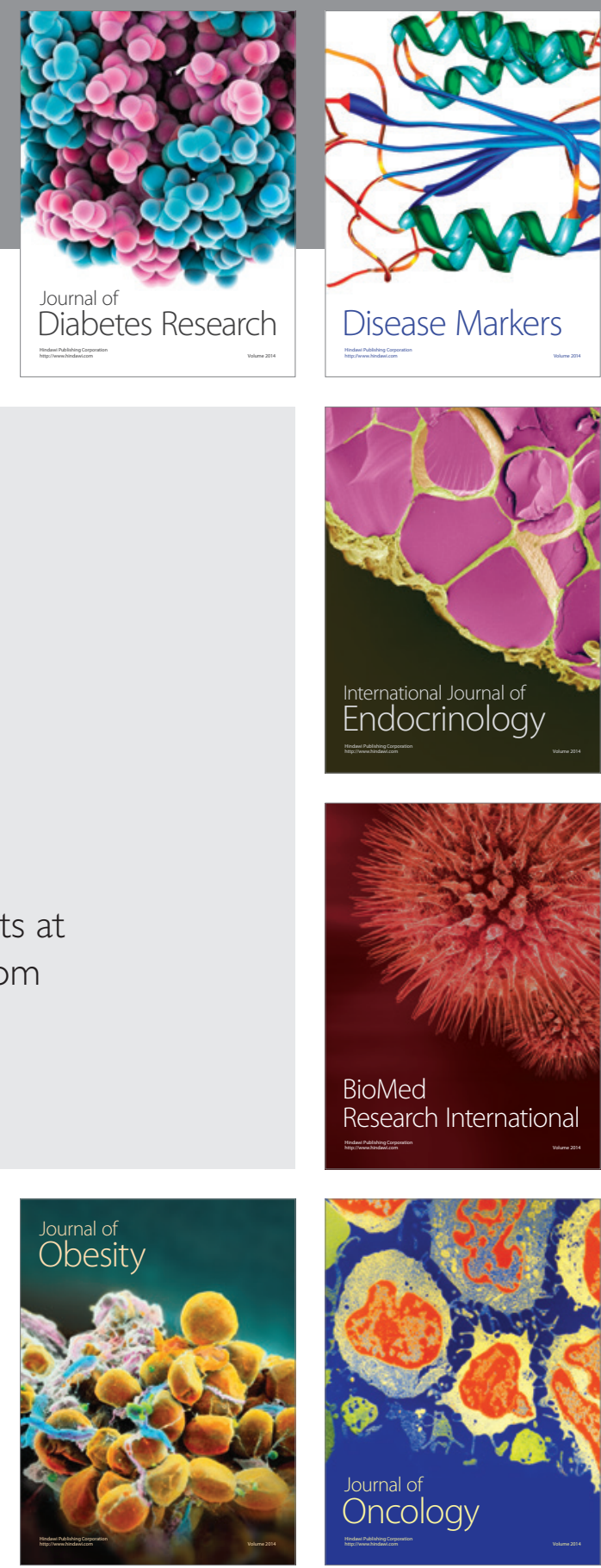

Disease Markers
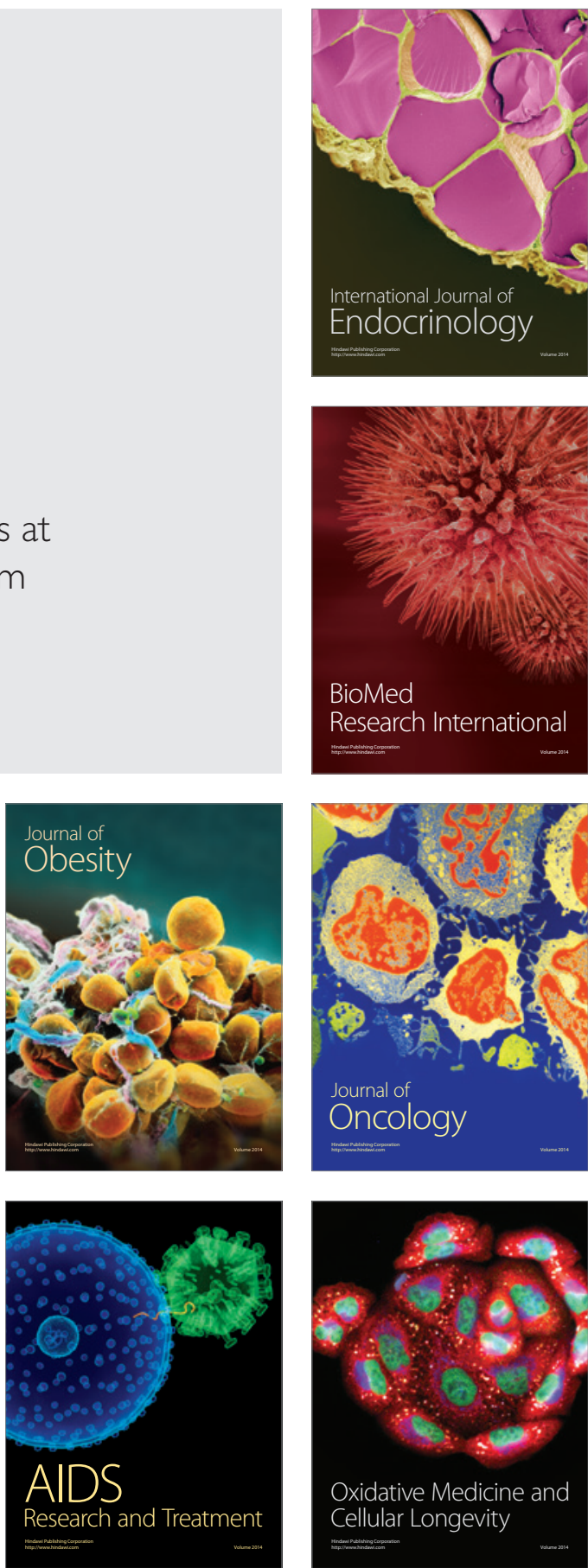